\title{
Feasibility Study of Automated Framework for Estimating Lung Tumor Locations for Target-Based Patient Positioning in Stereotactic Body Radiotherapy
}

\author{
Satoshi Yoshidome, ${ }^{1,2}$ Hidetaka Arimura, ${ }^{3}$ Katsumasa Nakamura, \\ Yoshiyuki Shioyama, ${ }^{4}$ Kazushige Atsumi, ${ }^{5}$ Yasuhiko Nakamura, ${ }^{6}$ Hideki Yoshikawa, ${ }^{6}$ \\ Kei Nishikawa, ${ }^{1}$ and Hideki Hirata ${ }^{3}$ \\ ${ }^{1}$ Department of Clinical Radiology, Kyushu University Beppu Hospital, 4546 Tsurumibaru, Tsurumi, Beppu 874-0838, Japan \\ ${ }^{2}$ Department of Health Sciences, Graduate School of Medical Sciences, Kyushu University, 3-1-1 Maidashi, Higashi-ku, \\ Fukuoka 812-8582, Japan \\ ${ }^{3}$ Department of Health Sciences, Faculty of Medical Sciences, Kyushu University, 3-1-1 Maidashi, Higashi-ku, Fukuoka 812-8582, Japan \\ ${ }^{4}$ Department of Clinical Radiology, Graduate School of Medical Sciences, Kyushu University, 3-1-1 Maidashi, Higashi-ku, \\ Fukuoka 812-8582, Japan \\ ${ }^{5}$ Department of Radiology, Kyushu University Beppu Hospital, 4546 Tsurumibaru, Tsurumi, Beppu 874-0838, Japan \\ ${ }^{6}$ Division of Radiology, Department of Medical Technology, Kyushu University Hospital, 3-1-1 Maidashi, Higashi-ku, \\ Fukuoka 812-8582, Japan
}

Correspondence should be addressed to Hidetaka Arimura; arimurah@med.kyushu-u.ac.jp

Received 27 August 2014; Revised 31 October 2014; Accepted 31 October 2014

Academic Editor: Noriyoshi Sawabata

Copyright (C) 2015 Satoshi Yoshidome et al. This is an open access article distributed under the Creative Commons Attribution License, which permits unrestricted use, distribution, and reproduction in any medium, provided the original work is properly cited.

Objective. To investigate the feasibility of an automated framework for estimating the lung tumor locations for tumor-based patient positioning with megavolt-cone-beam computed tomography (MV-CBCT) during stereotactic body radiotherapy (SBRT). Methods. A lung screening phantom and ten lung cancer cases with solid lung tumors, who were treated with SBRT, were employed to this study. The locations of tumors in MV-CBCT images were estimated using a tumor-template matching technique between a tumor template and the MV-CBCT. Tumor templates were produced by cropping the gross tumor volume (GTV) regions, which were enhanced by a Sobel filter or a blob structure enhancement (BSE) filter. Reference tumor locations (grand truth) were determined based on a consensus between a radiation oncologist and a medical physicist. Results. According to the results of the phantom study, the average Euclidean distances of the location errors in the original, Sobel-filtered, and BSE-filtered images were $2.0 \pm 4.1 \mathrm{~mm}, 12.8 \pm 9.4 \mathrm{~mm}$, and $0.4 \pm 0.5 \mathrm{~mm}$, respectively. For clinical cases, these were $3.4 \pm 7.1 \mathrm{~mm}, 7.2 \pm 11.6 \mathrm{~mm}$, and $1.6 \pm$ $1.2 \mathrm{~mm}$, respectively. Conclusion. The feasibility study suggests that our proposed framework based on the BSE filter may be a useful tool for tumor-based patient positioning in SBRT.

\section{Introduction}

Stereotactic body radiotherapy (SBRT) is employed for administering higher doses to ablate lung cancer while sparing surrounding normal tissues. In SBRT, a high dose of radiation, such as 12 Gy per session, is administered to a small localized region for small number of fractions [1-5]. The key to successful SBRT is the accurate patient positioning with immobilization devices and the use of image-guided positioning technologies using a two-dimensional (2D) electronic portal imaging device (EPID), 2D kilovolt- (kV-) imaging device, and/or three-dimensional (3D) $\mathrm{kV}$ - or megavolt(MV-) cone-beam computed tomography (CBCT), which are employed for acquiring "verification images" [6-11].

CBCT-based image-guided radiotherapy (IGRT) systems are widely used to increase the accuracy of the SBRT. 
TABLE 1: Patient characteristics for 10 cases used in this study.

\begin{tabular}{lccccc}
\hline Case number & Age & Gender & Tumor location & Number of fractions & Effective diameter $(\mathrm{mm})$ \\
\hline 1 & 89 & $\mathrm{M}$ & Lt. upper & 4 & 9.35 \\
2 & 75 & $\mathrm{M}$ & Lt. middle & 4 & 12.30 \\
3 & 72 & $\mathrm{~F}$ & Rt. middle & 4 & 11.94 \\
4 & 83 & $\mathrm{M}$ & Rt. upper & 4 & 10.07 \\
5 & 80 & $\mathrm{M}$ & Rt. upper & 4 & 12.91 \\
6 & 66 & $\mathrm{M}$ & Rt. lower & 4 & 5.35 \\
7 & 71 & M & Rt. lower & 4 & 5.90 \\
8 & 75 & F & Lt. upper & 4 & 5.38 \\
9 & 83 & F & Rt. upper & 4 & 13.12 \\
10 & 77 & M & Rt. lower & & 4 \\
\hline
\end{tabular}

${ }^{*} \mathrm{~F}$ : female and M: male.

However, many institutions use the following two-step IGRTbased procedure for the patient setup, that is, (1) the initial patient positioning based on bone structures and then (2) the manual and subjective translation of patients so that tumors' regions in the verification images align with those in the planning CT images $[12,13]$. This is because bone-based patient positioning leads to tumor misalignment between treatment planning CT images and pretreatment CBCT images, and it requires relatively larger safety margins because of the large interfractional baseline shifts and the deformity of the tumor [14-17]. If the patient positioning could be automatically and quantitatively performed by solely one-step procedure based on the tumor, it would result in better reproducibility of the patient setup for several fractions. Furthermore, if the tumor-based automated approach would accurately perform the patient setup, we could achieve high rates of local tumor control with low rates of toxicity [18-20] and would reduce the time required for patient positioning due to reduction of one step.

To the best of our knowledge, there have been no studies on frameworks for estimating lung tumor locations, which can be employed for automated patient positioning based on tumor regions. The goal of the present study was to investigate the feasibility of an automated framework for estimating the lung tumor locations for tumor-based patient positioning with MV-CBCT in SBRT.

\section{Methods and Materials}

2.1. Overall Design of Proposed Framework. Figure 1 presents the overall design of the proposed framework. In the proposed framework, the tumor location in an MV-CBCT image is estimated for each fraction using a tumor-template matching technique between the tumor template and MVCBCT image within the search region determined based on the gross tumor volume (GTV). The search region for the tumor location is determined based on the GTV region determined by a rigid registration between the planning CT and MV-CBCT images. Each tumor template is produced for a treatment course by cropping the GTV region, which is first enhanced by a Sobel filter for edge enhancement or by a blob structure enhancement (BSE) filter for tumor enhancement.

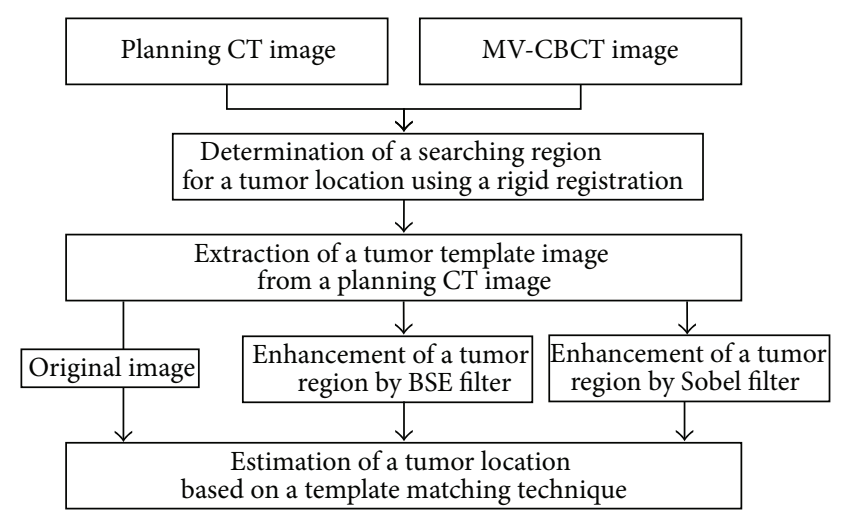

FIGURE 1: The overall design for estimating the lung tumor location in MV-CBCT images.

The tumor location is then estimated by the centroid of the GTV region in the MV-CBCT image using a tumor-template matching technique.

2.2. A Test Phantom. A lung screening phantom for CT (LSCT-001 type phantom, Kyoto Kagaku Co., Ltd., Kyoto, Japan) was employed for testing the proposed framework as validation test. Simulated tumors (CT value of lung: $-900 \mathrm{HU}$, contrast of CT value between lung and simulated tumor: $270 \mathrm{HU}$, sphere shape, and diameter: $10 \mathrm{~mm}$ ) were placed in apex pulmonis, tracheal bifurcation, and basis pulmonis of the lung screening phantom.

2.3. Clinical Cases. The institutional review board of our university hospital approved this retrospective study. Table 1 shows the patient characteristics of the cases included in this study. We selected 10 patients (age: 71-89 years, median: 76 years, seven males, and three females) with non-small-cell lung cancer, who were treated with SBRT from March 2010 to August 2011, based on selection criteria on tumor, that is, solitary, type (only solid), location in lung, and size. Eight tumors were solitary and two tumors were close to or attached to the lung wall. Five tumors were located in upper lung, 2 in middle lung, and 3 in lower lung. The mean effective 


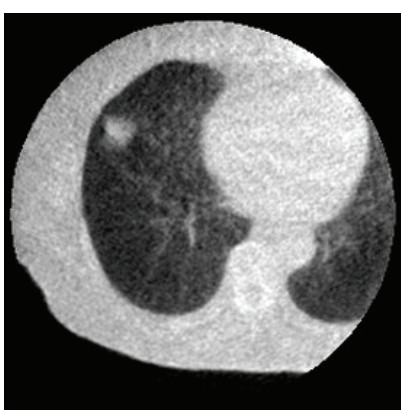

(a)

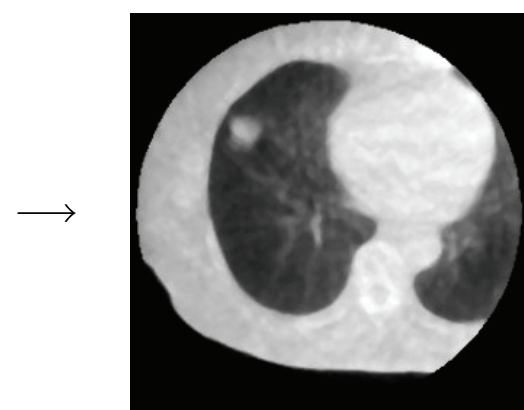

(b)

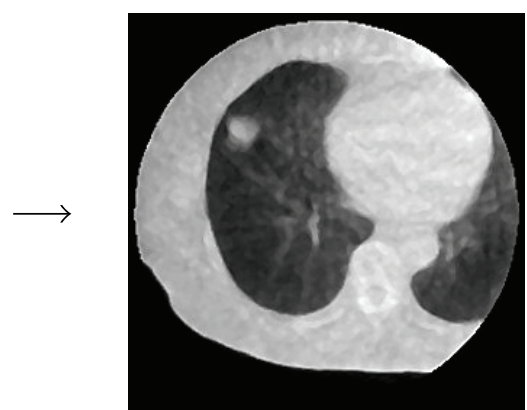

(c)

FIGURE 2: Illustrations of (a) an original MV-CBCT image, (b) a noise-reduced MV-CBCT image obtained using a median filter, and (c) an edge-enhanced MV-CBCT image obtained using a Laplacian of Gaussian (LoG) filter.

diameter of the 10 tumors was $10.43 \pm 2.93 \mathrm{~mm}$ (range: 5.38-14.35 mm). Four MV-CBCT images of the patients were acquired for four fractions for patient positioning at a linear accelerator (ONCOR Impression plus, Siemens AG, Berlin and Munich, Germany) with an accelerating voltage of $4 \mathrm{MV}$. An MV-CBCT imaging was performed for initial patient positioning in each fraction, but not repositioning between beams. The total prescribed dose for each patient was $48 \mathrm{~Gy}$ $(12 \mathrm{~Gy} /$ fraction) at the isocenter for four fractions.

2.4. Planning CT and CBCT Images. Planning CT images were acquired from a 64-slice CT scanner (SOMATOM Sensation 64, Siemens, Munich), whose CT images had an axial field of view (FOV) of $500 \mathrm{~mm} \times 500 \mathrm{~mm}$, a matrix size of $512 \times 512$, a pixel size of $0.9766 \mathrm{~mm}$, a slice thickness of $1.024 \mathrm{~mm}$, and a bit depth of 12. The number of planning CT images ranged from 156 to 224 .

MV-CBCT images were taken by using a CBCT imaging system (MVision, Siemens, Munich) including an EPID (OPTIVUE 2.0, Siemens, Munich).

The CBCT imaging system collects projection images on the EPID and reconstructs the CBCT images (a FOV of $274 \mathrm{~mm} \times 274 \mathrm{~mm} \times 274 \mathrm{~mm}$, a matrix size of $256 \times 256$, a pixel size of $1.0703 \mathrm{~mm}$, a slice thickness of $1.0 \mathrm{~mm}$, and a bit depth of 12).

The original planning CT images and the MV-CBCT images were converted to images with a $1.0 \mathrm{~mm}$ isotropic voxel size using a 3D tricubic interpolation technique. The isotropic images were processed by a median filter (filter size: $3 \times 3 \times 3$ ) for reducing image noise and by a Laplacian of Gaussian (LoG) filter (standard deviation: 0.7 pixels) for edge enhancement, because the MV-CBCT images had image noise and blurred target edges due to Compton scattered Xrays and high energy X-rays. Figure 2 shows an original MVCBCT image (a), a noise-reduced image by a median filter (b), and an edge-enhanced image by a LoG filter (c). The image noise was reduced, and the target edge was slightly enhanced.

2.5. Determination of a Region to Search for the Tumor Location in $M V$-CBCT Images. The region to search for the tumor location was determined based on the GTV region determined by a rigid registration between the planning CT and MV-CBCT images. First, planning CT and MV-CBCT images were transformed by using a 3D linear interpolation technique to $5.0 \mathrm{~mm}$ isotropic images in order to reduce the calculation time for the registration. Second, the rigid registration was performed by finding the location with the maximum cross correlation coefficient between the planning $\mathrm{CT}$ and MV-CBCT images according to the following equation [21]:

$$
\begin{aligned}
& \quad\left(x_{\max }, y_{\max }, z_{\max }\right)=\arg \max _{x, y, z}(C(x, y, z)) \\
& C(x, y, z) \\
& =\frac{1}{L M N} \\
& \times \sum_{i=0}^{L-1} \sum_{j=0}^{M-1} \sum_{k=0}^{N-1} \frac{(t(i, j, k)-\bar{t})(f(x+i, y+j, z+k)-\bar{f})}{\sigma_{t} \cdot \sigma_{f}},
\end{aligned}
$$

where $C(x, y, z)$ is the cross correlation coefficient at a coordinate $(x, y, z)$ on a planning CT image, $L, M, N$ are the numbers of pixels in the $x, y$, and $z$ directions in an MV-CBCT image, respectively, $t(i, j, k)$ is the pixel value at a coordinate $(i, j, k)$ on an MV-CBCT image, $f(x, y, z)$ is the pixel value at a coordinate $(x, y, z)$ on a planning CT image, $\bar{t}$ and $\sigma_{t}$ are the mean and standard deviation of pixel values in an MV-CBCT image, and $\bar{f}$ and $\sigma_{f}$ are the mean and standard deviation of pixel values in a planning CT image. The ranges of $x, y$, and $z$ coordinates, $R_{x}, R_{y}, R_{z}$, are determined by the following equation:

$$
\begin{aligned}
& R_{x}=X-L \\
& R_{y}=Y-M \\
& R_{z}=Z-N,
\end{aligned}
$$

where $X, Y, Z$ are the numbers of pixels in the $x, y$, and $z$ directions of a planning CT image. Please note that the cross correlation coefficient was calculated within an overlapped region between the planning CT and MV-CBCT images. 


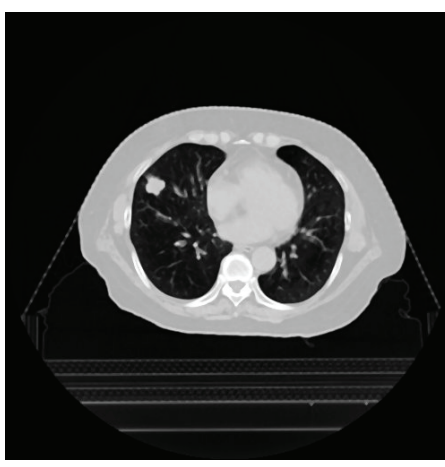

(a)

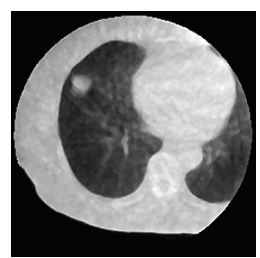

(b)

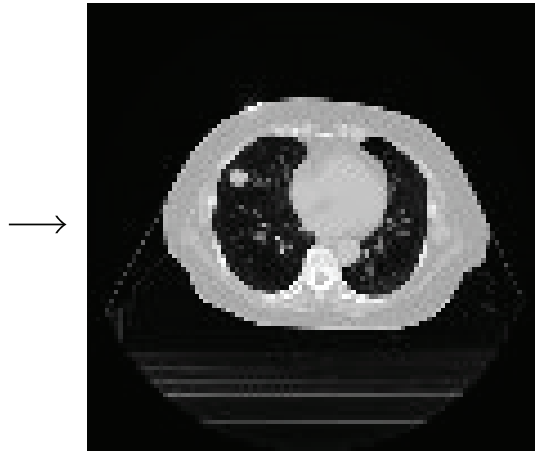

(c)

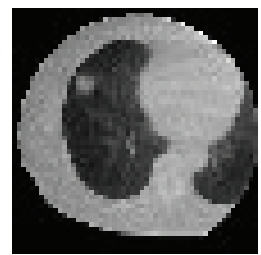

(d)

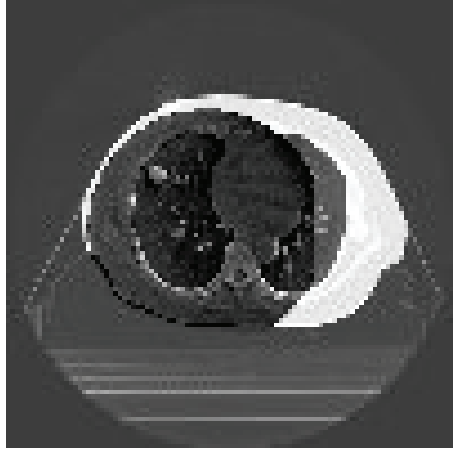

(e)

FIGURE 3: Illustrations of (a) a $1.0 \mathrm{~mm}$ isotropic planning CT image, (b) an MV-CBCT image, (c) a $5.0 \mathrm{~mm}$ isotropic planning CT image, (d) an MV-CBCT image, and (e) a subtraction image between a planning CT image and an MV-CBCT image after the rigid registration.

The overlapped region between the planning CT and MVCBCT images is always the same as the matrix size of the MVCBCT image, because all MV-CBCT images are smaller than the planning CT images. Figure 3 shows $1.0 \mathrm{~mm}$ isotropic planning CT (a) and MV-CBCT images (b), $5.0 \mathrm{~mm}$ isotropic planning CT (c) and MV-CBCT images (d), and a subtraction image (e) between the planning CT and MV-CBCT images after the rigid registration.

Third, the $5.0 \mathrm{~mm}$ isotropic planning CT and MV-CBCT images were converted again to $1.0 \mathrm{~mm}$ isotropic images using the same interpolation method. After this step, only $1.0 \mathrm{~mm}$ isotropic images were used for all the following processes. Fourth, the centroid of a GTV region in the MV-CBCT image was placed at the isocenter after the rigid registration. Finally, a circumscribing cuboid of the GTV region dilated by 20 pixels in each coordinate direction was derived as the region to search for the tumor location. The dilation processing was performed to 26 neighbors of a center pixel.

2.6. Extraction of a Tumor Template from Planning CT Images. Tumor templates were extracted by cropping a circumscribing cuboid of the GTV from the $1.0 \mathrm{~mm}$ original planning CT image. These templates were referred to as "planning CT tumor templates." The coordinates of isocenters and contour data of GTVs were obtained from DICOM-RT structure sets, which were produced on a radiation treatment planning (RTP) system (XiO, Elekta, Stockholm).

2.7. Enhancement of the Tumor Region in Planning CT Tumor Templates and MV-CBCT Images. Planning CT tumor templates and $\mathrm{MV}$-CBCT images were processed for the enhancement of tumors by using a Sobel filter or a blob structure enhancement (BSE) filter. The Sobel filter with a filter size of $3 \times 3 \times 3$ pixels was used to enhance the tumor edges.

The BSE filter can selectively enhance spherical structures [22], such as lung tumors, based on eigenvalues of the following Hessian matrix:

$$
\begin{array}{r}
H(\mathbf{p}, \sigma)=\left[\begin{array}{ccc}
I_{x x}(\mathbf{p}, \sigma) & I_{x y}(\mathbf{p}, \sigma) & I_{x z}(\mathbf{p}, \sigma) \\
I_{y x}(\mathbf{p}, \sigma) & I_{y y}(\mathbf{p}, \sigma) & I_{y x}(\mathbf{p}, \sigma) \\
I_{z x}(\mathbf{p}, \sigma) & I_{z y}(\mathbf{p}, \sigma) & I_{z z}(\mathbf{p}, \sigma)
\end{array}\right] \\
I_{x^{i} y^{j} z^{k}}(\mathbf{p}, \sigma)=\left\{\frac{\partial^{2}}{\partial x^{i} \partial y^{j} \partial z^{k}} G(\mathbf{p}, \sigma)\right\} * I(\mathbf{p}) \\
i+j+k=2,
\end{array}
$$

where $H(\mathbf{p}, \sigma)$ is the Hessian matrix at a position vector $\mathbf{p}=$ $(x, y, z)$ and $G(\mathbf{p}, \sigma)$ is the Gaussian function with a standard deviation $\sigma$. The BSE filter was defined by

$$
\begin{gathered}
F\left(\lambda_{1}, \lambda_{2}, \lambda_{3}\right) \\
= \begin{cases}\left|\lambda_{3}\right| \psi\left(\lambda_{2}, \lambda_{3}\right) \psi\left(\lambda_{1}, \lambda_{2}\right) & \text { if } \lambda_{3} \leq \lambda_{2} \leq \lambda_{1} \leq 0 \\
0 & \text { otherwise }\end{cases} \\
\psi\left(\lambda_{s}, \lambda_{t}\right)= \begin{cases}\left(\frac{\lambda_{s}}{\lambda_{t}}\right)^{\gamma} & \text { if } \lambda_{s} \leq \lambda_{t}<0 \\
0 & \text { otherwise, }\end{cases}
\end{gathered}
$$

where $\lambda_{1}, \lambda_{2}, \lambda_{3}$ were the first, second, and third eigenvalues of the Hessian matrix $H(\mathbf{p}, \sigma)$, respectively, $\psi$ was the weight 
function, and $\gamma$ was the control factor for the sharpness of selectivity for the conditions of the local structure. In this study, the standard deviation, $\sigma$, was set as the quarter of the effective diameter of a GTV region, and $\gamma$ was set as 1.0. These parameters were empirically determined so that the tumors were well enhanced based on a consensus between a radiation oncologist and a medical physicist. The effective diameter, $d$, for the GTV region was calculated by the following equation:

$$
d=2 \sqrt[3]{\frac{3 V_{\mathrm{GTV}}}{4 \pi}}
$$

where $V_{\mathrm{GTV}}$ was the GTV produced on a RTP system $(\mathrm{XiO}$, Elekta, Stockholm).

\subsection{Estimation of Tumor Locations Based on a Template} Matching Technique. A tumor's location was estimated based on template matching between a planning CT tumor template and an MV-CBCT image within the search region. The template matching was performed by determining the location with the maximum cross correlation coefficient between the planning CT tumor template and MV-CBCT image according to (1). After the template matching technique was applied, the GTV region was placed in the MV-CBCT image, and the centroid of the binarized GTV region was considered to be the tumor location. The binarized GTV region was obtained by using an Otsu's thresholding method [23]. The centroid $\left(x_{c}, y_{c}, z_{c}\right)$ of the GTV region was calculated by following equation:

$$
\left(x_{c}, y_{c}, z_{c}\right)=\left(\frac{1}{T}\left(\sum_{i=0}^{T} x_{i}\right), \frac{1}{T}\left(\sum_{i=0}^{T} y_{i}\right), \frac{1}{T}\left(\sum_{i=0}^{T} z_{i}\right)\right),
$$

where $\left(x_{i}, y_{i}, z_{i}\right)$ was a coordinate of a pixel in the binarized GTV region and $T$ was the number of pixels in the GTV region.

2.9. Evaluation of the Proposed Framework. The reference tumor region in each $\mathrm{MV}$-CBCT image was determined based on a consensus between a radiation oncologist and a medical physicist. The centroid of the reference tumor region in an MV-CBCT image was considered to be the reference tumor location (grand truth). The proposed framework was evaluated by measuring location errors between the reference tumor location and the tumor location estimated by the proposed framework in three directions (left-right, anterior-posterior, and superior-interior) and its corresponding Euclidean distance. The average and the standard deviation of location errors were analyzed by using Student $t$-test and $F$-test, respectively.

\section{Results}

Figure 4 shows an MV-CBCT image (a), a search region extracted from the MV-CBCT image (b), an edge-enhanced image with the Sobel filter in the search region (c), and a spherical shape-enhanced image obtained using the BSE filter in the search region (d). Figure 5 shows a planning CT image (a), a planning CT tumor template (b), an edge-enhanced tumor template with a Sobel filter (c), and a spherical shapeenhanced tumor template obtained using a BSE filter (d). The tumor appearance in the MV-CBCT image was blurred due to high-energy $\mathrm{X}$-rays and scattered $\mathrm{X}$-rays, compared with that in the planning CT image. The degree of the tumor edge enhancement with the Sobel filter in the MV-CBCT image shown in Figure 4(c) was weaker than that in the planning CT tumor template shown in Figure 5(c). In contrast, the strength of the tumor enhancement with the BSE filter in the MV-CBCT image shown in Figure 4(d) was comparable with that in the planning CT tumor template shown in Figure 5(d).

Figure 6 shows an MV-CBCT image with a GTV region determined by the proposed framework (a), an original GTV region and its outline (white solid line) cropped from an MVCBCT image (b), a binarized GTV region indicated in gray (c), and the original GTV region with an estimated tumor location indicated by $\mathrm{x}(\mathrm{d})$. The tumor location indicated by $\mathrm{x}$ (d) was estimated as the centroid of the GTV region indicated in gray (c), as shown in Figure 6.

According to the results of the phantom study, the average Euclidean distances of the location errors in the original image, Sobel-filtered images, and BSE-filtered images were $2.0 \pm 4.1 \mathrm{~mm}, 12.8 \pm 9.4 \mathrm{~mm}$, and $0.4 \pm 0.5 \mathrm{~mm}$, respectively. The proposed framework using the BSE filter can estimate the tumor locations with the errors less than $1 \mathrm{~mm}$ in all locations in the lung.

Figure 7 shows the location errors estimated by the proposed framework using three different types of planning CT tumor templates, that is, original images, Sobel-filtered images, and BSE-filtered images. The average Euclidean distances of the location errors in the original image templates, Sobel-filtered templates, and BSE-filtered templates were 3.4 $\pm 7.1 \mathrm{~mm}, 7.2 \pm 11.6 \mathrm{~mm}$, and $1.6 \pm 1.2 \mathrm{~mm}$, respectively. The means of the location errors with the two filtered templates had no statistically significant difference with the original image templates $(P>0.05)$. However, the estimation of the tumor locations obtained using BSE filtered templates was significantly more accurate on average compared with the Sobel-filtered templates $(P<0.05)$. In addition, the standard deviations of the location errors with the BSE-filtered templates were significantly smallest in all three directions and the Euclidean distance among the three templates $(P<$ 0.05). These results on the standard deviations of the location errors mean that the precision of the proposed framework using the BSE-filtered templates was highest among the three templates.

\section{Discussion}

Guckenberger et al. reported that the intraobserver and interobserver variability of the subjective evaluation of lung tumor location errors were $0.9 \pm 0.8 \mathrm{~mm}$ (maximum $3.5 \mathrm{~mm}$ ) and $2.3 \pm 1.1 \mathrm{~mm}$ (maximum $4.4 \mathrm{~mm}$ ), respectively, in SBRT when using a CBCT-based IGRT [24]. On the other hand, the average Euclidean distance of tumor errors in the lung screening phantom was $4.84 \pm 1.45 \mathrm{~mm}$, which was measured by using an image guided patient positioning (IGPP) system in the radiotherapy system used for this study. The location 


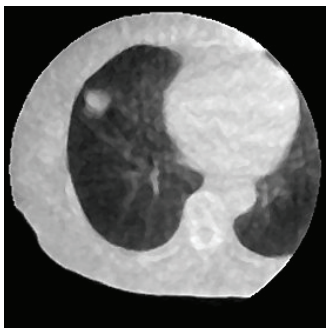

(a)

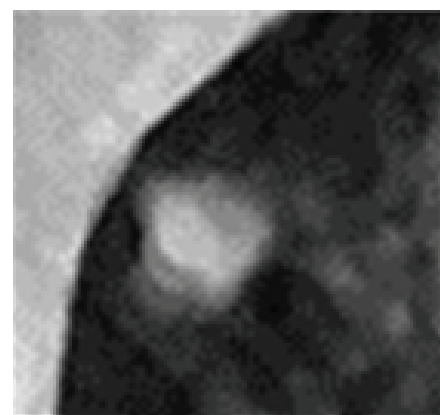

(b)

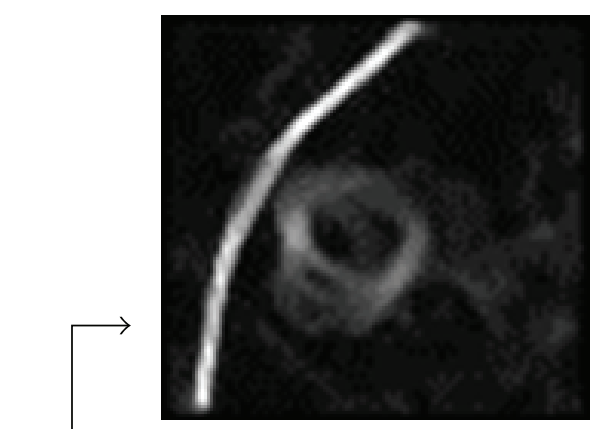

(c)

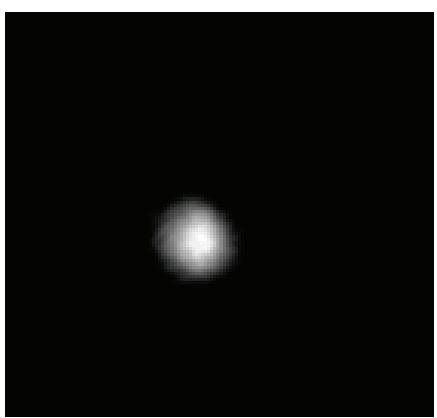

(d)

FIgURE 4: Illustrations of (a) an MV-CBCT image, (b) a search region extracted from the MV-CBCT image, (c) an edge-enhanced image obtained using the Sobel filter in the search region, and (d) a tumor-enhanced image obtained using the BSE filter in the search region.

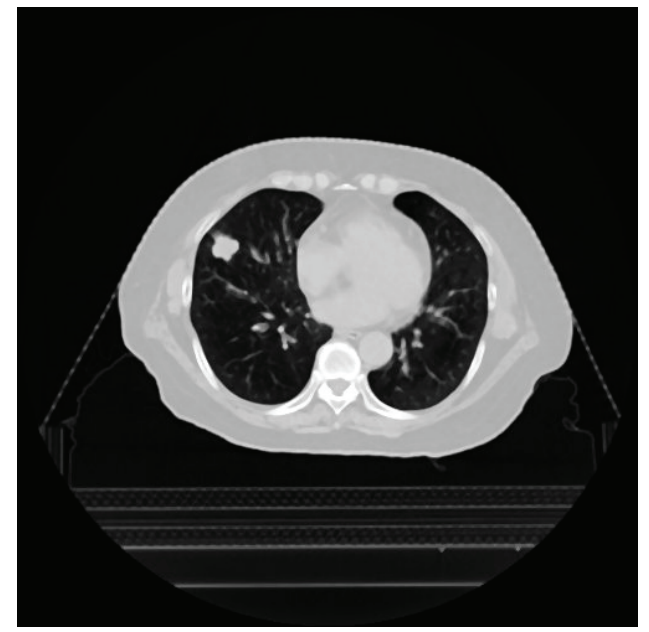

(a)

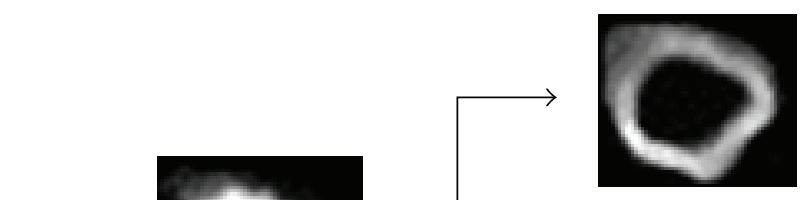

(c)

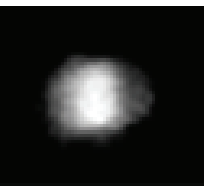

(d)

FIGURE 5: Illustrations of (a) a planning CT image, (b) a planning CT tumor template, (c) an edge-enhanced tumor template obtained using a Sobel filter, and (d) a spherical shape-enhanced tumor template obtained using a BSE filter.

errors $(1.6 \pm 1.2 \mathrm{~mm})$ obtained by the proposed framework based on the BSE filter were therefore smaller than those by the IGPP system as well as the interobserver variability, but larger than the intraobserver variability.

The systematic and random errors [25, 26] of manual patient positioning measured for 36 lung cancer patients, which were chosen based on the same selection criteria as the 10 test cases used for this study, were $3.53 \mathrm{~mm}$ and $4.01 \mathrm{~mm}$, respectively. On the other hand, the accuracy and precision of the proposed automated framework were $1.6 \mathrm{~mm}$ and $1.2 \mathrm{~mm}$, respectively, which were smaller than those in the manual method, although the accuracy and precision of manual patient positioning depend on the institution.

In principle, the proposed framework can be applied to $\mathrm{kV}-\mathrm{CBCT}$ as well as MV-CBCT. The soft tissue contrast in $\mathrm{kV}$-CBCT images is higher than that in MV-CBCT images 


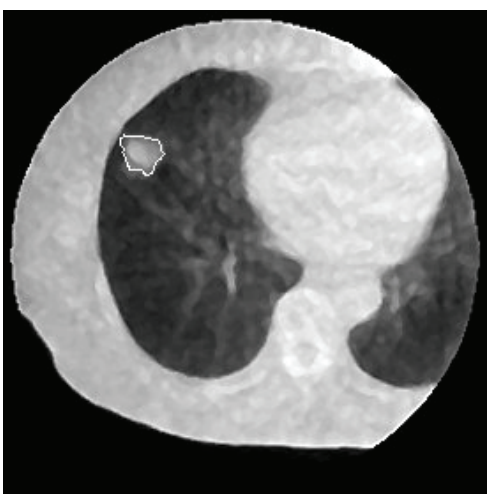

(a)

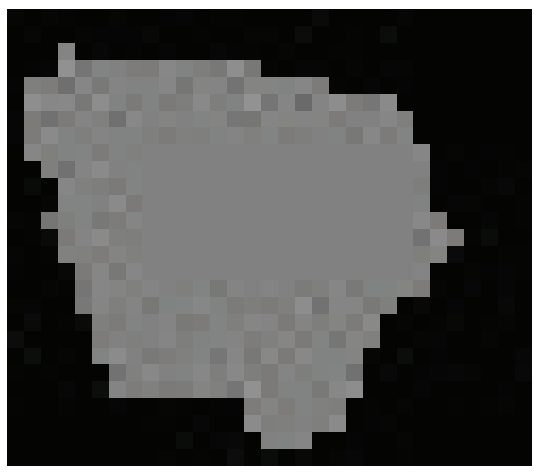

(c)

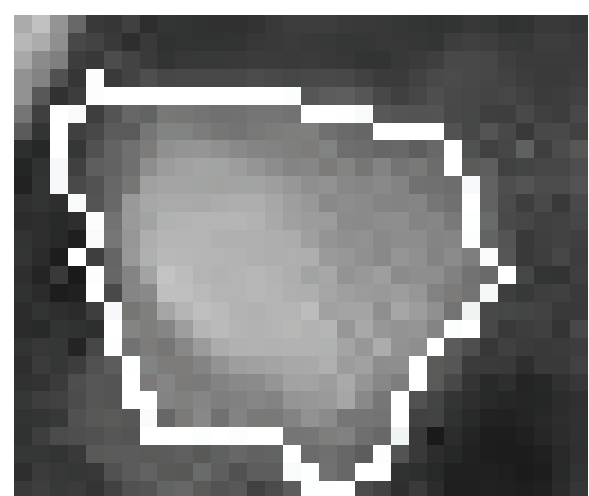

(b)

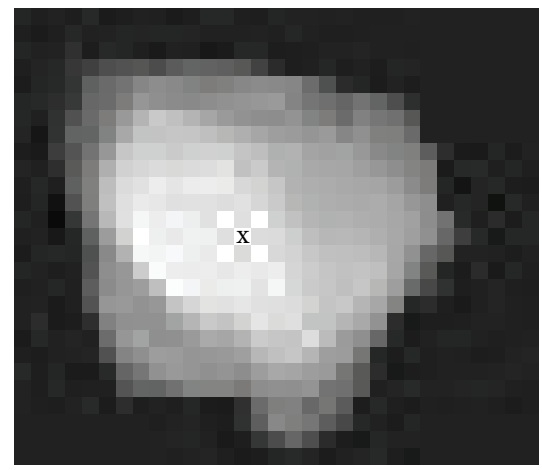

(d)

FIgURE 6: Illustrations of (a) an MV-CBCT image with a GTV region determined by the proposed framework, (b) a GTV region cropped from an MV-CBCT image, (c) a binarized GTV region indicated in gray, and (d) a tumor location indicated by $\mathrm{x}$.

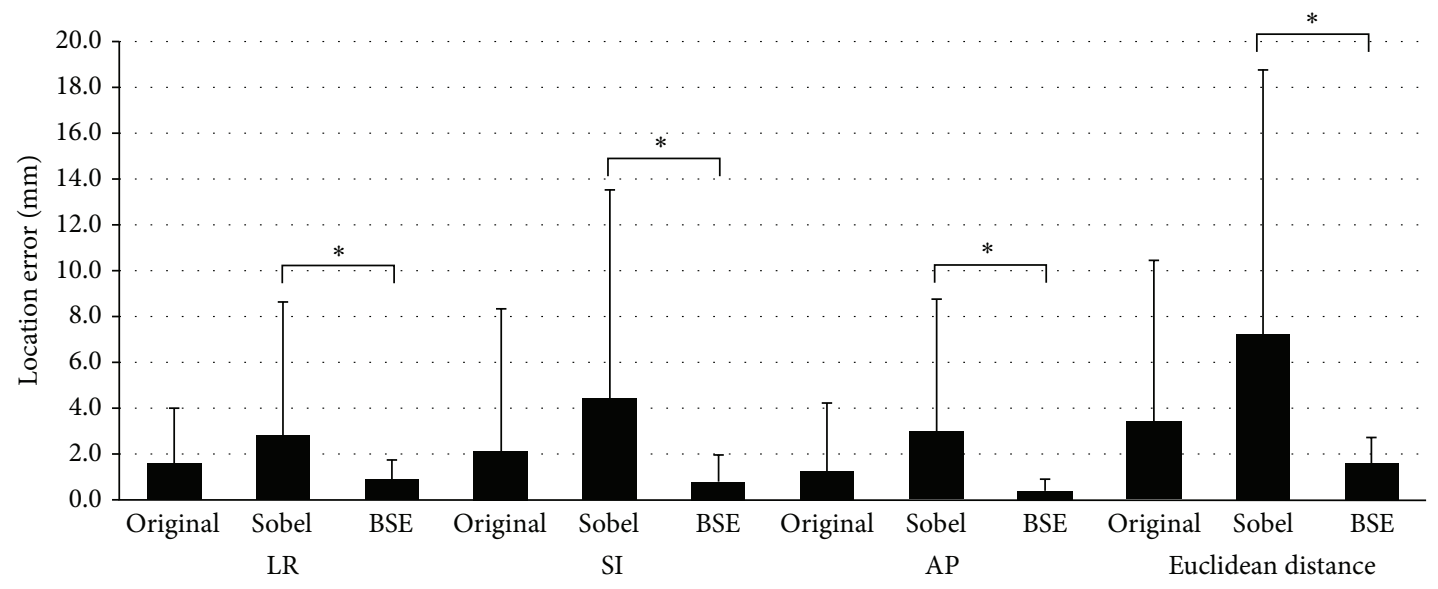

LR: left-right, SI: superior-inferior, AP: anterior-posterior

$t$-test: ${ }^{*} P<0.05$

F-test: $P<0.05$ (all combinations)

FIGURE 7: The results of a comparison of the location errors estimated by the proposed framework using three different types of planning CT tumor templates, that is, original images, Sobel-filtered images, and BSE-filtered images. 
[27]. Therefore, the results obtained using $\mathrm{kV}-\mathrm{CBCT}$ images may be better than the results obtained using MV-CBCT images. Furthermore, additional patient dose at the $\mathrm{kV}$ CBCT imaging could be less than that at the MV-CBCT imaging, because the mean dose of the simulated tumor in the lung screening phantom was $5.8 \mathrm{cGy}$ at the MV-CBCT imaging used in this study, which was larger than D50 of 0.2 to $0.42 \mathrm{cGy}$ to the heart for representative thorax images at the $\mathrm{kV}-\mathrm{CBCT}$ imaging reported by Ding and Munro [28].

The proposed framework has two major limitations: the calculation time and the limited types of cases. The average and maximum processing time of the proposed framework were 40 minutes and 58 seconds and 72 minutes and 12 seconds, respectively. This limitation may be overcome by implementing the proposed framework with general purpose computing on graphics processing units (GPGPU) or parallel computing techniques on computer clusters. The second limitation is that the proposed framework was applied only to solid lung cancer cases. Therefore, the proposed framework needs to be applied to large databases, including cases with various types of lung cancers, such as ground-glass opacity (GGO), and cases with diseases such as pneumonia, in order to determine whether it can also be applicable to such cases.

\section{Conclusion}

We have proposed an automated framework for estimating the lung tumor locations for tumor-based patient positioning with MV-CBCT during SBRT. The proposed framework with the BSE filter could automatically estimate the lung tumor locations with errors less than $1 \mathrm{~mm}$ for a lung screening phantom and $2 \mathrm{~mm}$ for clinical cases. If the calculation time of the proposed framework was improved, the proposed framework based on the BSE filtered image might be one of the tools for tumor-based patient positioning in SBRT.

\section{Conflict of Interests}

The authors declare that there is no conflict of interests regarding the publication of this paper.

\section{Acknowledgments}

The authors would like to express their appreciation to all of the members of the Arimura Laboratory who provided them with their precious comments and advice and who made great contributions to this study. This research was partially supported by the JSPS KAKENHI Grant no. 26670301 (Grantin-Aid for Challenging Exploratory Research).

\section{References}

[1] S. H. Benedict, K. M. Yenice, D. Followill et al., "Stereotactic body radiation therapy: the report of AAPM Task Group 101," Medical Physics, vol. 37, no. 8, pp. 4078-4101, 2010.

[2] H. Shirato, Y. Seppenwoolde, K. Kitamura, R. Onimura, and S. Shimizu, "Intrafractional tumor motion: lung and liver," Seminars in Radiation Oncology, vol. 14, no. 1, pp. 10-18, 2004.
[3] J. Wulf, U. Hädinger, U. Oppitz, W. Thiele, and M. Flentje, "Impact of target reproducibility on tumor dose in stereotactic radiotherapy of targets in the lung and liver," Radiotherapy \& Oncology, vol. 66, no. 2, pp. 141-150, 2003.

[4] K. Takayama, Y. Nagata, Y. Negoro et al., "Treatment planning of stereotactic radiotherapy for solitary lung tumor," International Journal of Radiation Oncology Biology Physics, vol. 61, no. 5, pp. 1565-1571, 2005.

[5] D. H. Lim, B. Y. Yi, A. Mirmiran, A. Dhople, M. Suntharalingam, and W. D. D'Souza, "Optimal beam arrangement for stereotactic body radiation therapy delivery in lung tumors," Acta Oncologica, vol. 49, no. 2, pp. 219-224, 2010.

[6] M. Høyer, "Improved accuracy and outcome in radiotherapy of lung cancer," Radiotherapy and Oncology, vol. 87, no. 1, pp. 1-2, 2008.

[7] M. Fuss, J. Boda-Heggemann, N. Papanikolau, and B. J. Salter, "Image-guidance for stereotactic body radiation therapy," Medical Dosimetry, vol. 32, no. 2, pp. 102-110, 2007.

[8] Y. Nagata, Y. Negoro, T. Aoki et al., "Clinical outcomes of 3D conformal hypofractionated single high-dose radiotherapy for one or two lung tumors using a stereotactic body frame," International Journal of Radiation Oncology, Biology, Physics, vol. 52, no. 4, pp. 1041-1046, 2002.

[9] Y. Negoro, Y. Nagata, T. Aoki et al., "The effectiveness of an immobilization device in conformal radiotherapy for lung tumor: reduction of respiratory tumor movement and evaluation of the daily setup accuracy," International Journal of Radiation Oncology Biology Physics, vol. 50, no. 4, pp. 889-898, 2001.

[10] K. Nakagawa, Y. Aoki, M. Tago, A. Terahara, and K. Ohtomo, "Megavoltage CT-assisted stereotactic radiosurgery for thoracic tumors: original research in the treatment of thoracic neoplasms," International Journal of Radiation Oncology, Biology, Physics, vol. 48, no. 2, pp. 449-457, 2000.

[11] J. Zhou, B. Uhl, K. Dewitt et al., "Image-guided stereotactic body radiotherapy for lung tumors using BodyLoc with tomotherapy: clinical implementation and set-up accuracy," Medical Dosimetry, vol. 35, no. 1, pp. 12-18, 2010.

[12] J. P. Bissonnette, T. G. Purdie, J. A. Higgins, W. Li, and A. Bezjak, "Cone-Beam computed tomographic image guidance for lung cancer radiation therapy," International Journal of Radiation Oncology Biology Physics, vol. 73, no. 3, pp. 927-934, 2009.

[13] N. D. Richmond, K. E. Pilling, C. Peedell, D. Shakespeare, and C. P. Walker, "Positioning accuracy for lung stereotactic body radiotherapy patients determined by on-treatment cone-beam CT imaging," The British Journal of Radiology, vol. 85, no. 1014, pp. 819-823, 2012.

[14] M. Guckenberger, T. Krieger, A. Richter et al., "Potential of image-guidance, gating and real-time tracking to improve accuracy in pulmonary stereotactic body radiotherapy," Radiotherapy and Oncology, vol. 91, no. 3, pp. 288-295, 2009.

[15] M. Guckenberger, J. Meyer, J. Wilbert et al., "Intra-fractional uncertainties in cone-beam CT based image-guided radiotherapy (IGRT) of pulmonary tumors," Radiotherapy \& Oncology, vol. 83, no. 1, pp. 57-64, 2007.

[16] L. Masi, F. Casamassima, C. Menichelli et al., "On-line image guidance for frameless stereotactic radiotherapy of lung malignancies by cone beam CT: comparison between target localization and alignment on bony anatomy," Acta Oncologica, vol. 47, no. 7, pp. 1422-1431, 2008.

[17] W. Takahashi, H. Yamashita, S. Kida et al., "Verification of planning target volume settings in volumetric modulated arc 
therapy for stereotactic body radiation therapy by using intreatment 4-dimensional cone beam computed tomography," International Journal of Radiation Oncology, Biology, Physics, vol. 86, no. 3, pp. 426-431, 2013.

[18] J. Boda-Heggemann, F. Lohr, F. Wenz, M. Flentje, and M. Guckenberger, "kV cone-beam CT-based IGRT: a clinical review," Strahlentherapie und Onkologie, vol. 187, no. 5, pp. 284-291, 2011.

[19] J. J. Nuyttens and M. van de Pol, “The CyberKnife radiosurgery system for lung cancer," Expert Review of Medical Devices, vol. 9, no. 5, pp. 465-475, 2012.

[20] W. Li, A. Sahgal, M. Foote, B.-A. Millar, D. A. Jaffray, and D. Letourneau, "Impact of immobilization on intrafraction motion for spine stereotactic body radiotherapy using cone beam computed tomography," International Journal of Radiation Oncology Biology Physics, vol. 84, no. 2, pp. 520-526, 2012.

[21] H. Arimura, S. Katsuragawa, Q. Li, T. Ishida, and K. Doi, "Development of a computerized method for identifying the posteroanterior and lateral views of chest radiographs by use of a template matching technique," Medical Physics, vol. 29, no. 7, pp. 1556-1561, 2002.

[22] Y. Sato, S. Nakajima, N. Shiraga et al., "Three-dimensional multi-scale line filter for segmentation and visualization of curvilinear structures in medical images," Medical Image Analysis, vol. 2, no. 2, pp. 143-168, 1998.

[23] N. Otsu, "A threshold selection method from gray-level histograms," IEEE Transations Systems, Man, and Cybernetics, vol. 9, no. 1, pp. 62-66, 1979.

[24] M. Guckenberger, J. Meyer, J. Wilbert et al., "Cone-beam CT based image-guidance for extracranial stereotactic radiotherapy of intrapulmonary tumors," Acta Oncologica, vol. 45, no. 7, pp. 897-906, 2006.

[25] J. C. Stroom, H. C. J. de Boer, H. Huizenga, and A. G. Visser, "Inclusion of geometrical uncertainties in radiotherapy treatment planning by means of coverage probability," International Journal of Radiation Oncology, Biology, Physics, vol. 43, no. 4, pp. 905-919, 1999.

[26] M. van Herk, P. Remeijer, C. Rasch, and J. V. Lebesque, "The probability of correct target dosage: dose-population histograms for deriving treatment margins in radiotherapy," International Journal of Radiation Oncology Biology Physics, vol. 47, no. 4, pp. 1121-1135, 2000.

[27] W. W. K. Fung and V. W. C. Wu, "Image-guided radiation therapy using computed tomography in radiotherapy," Journal of Radiotherapy in Practice, vol. 10, no. 2, pp. 121-136, 2011.

[28] G. X. Ding and P. Munro, "Radiation exposure to patients from image guidance procedures and techniques to reduce the imaging dose," Radiotherapy and Oncology, vol. 108, no. 1, pp. 91-98, 2013. 


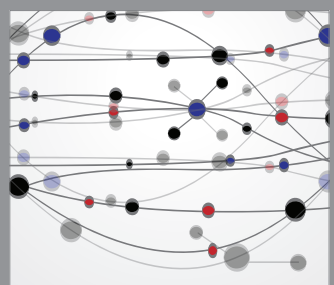

The Scientific World Journal
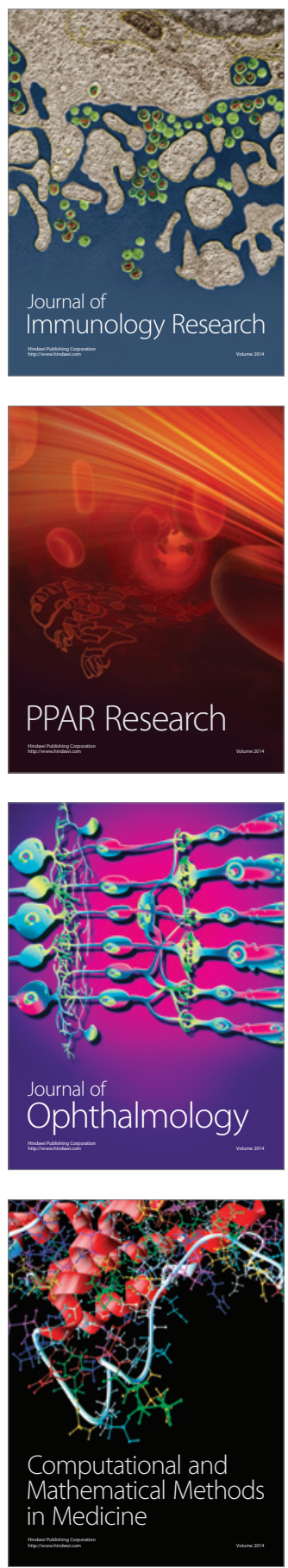

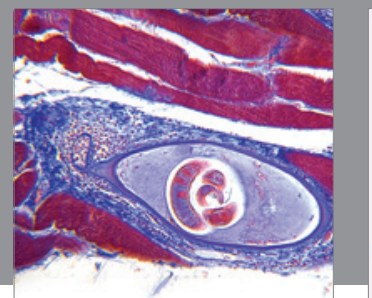

Gastroenterology

Research and Practice
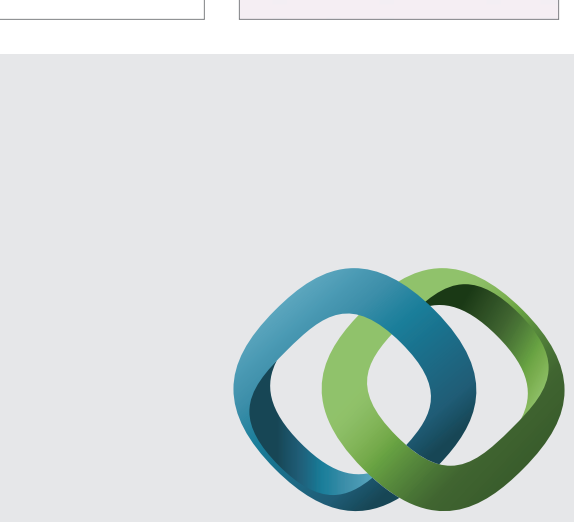

\section{Hindawi}

Submit your manuscripts at

http://www.hindawi.com
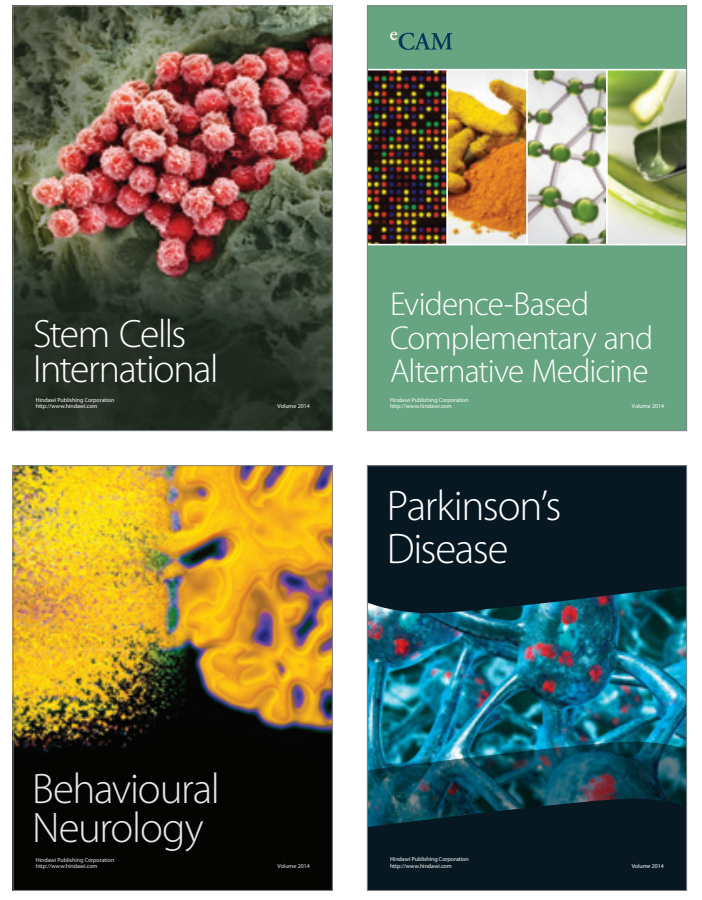
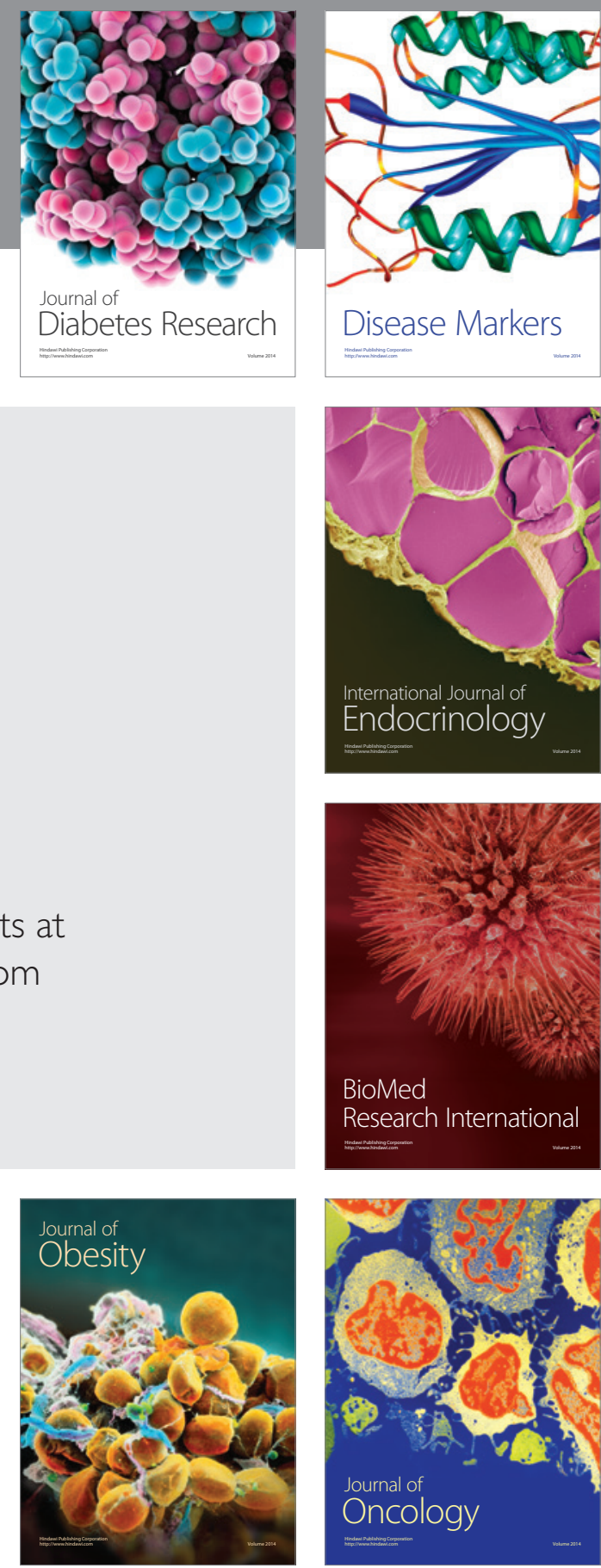

Disease Markers
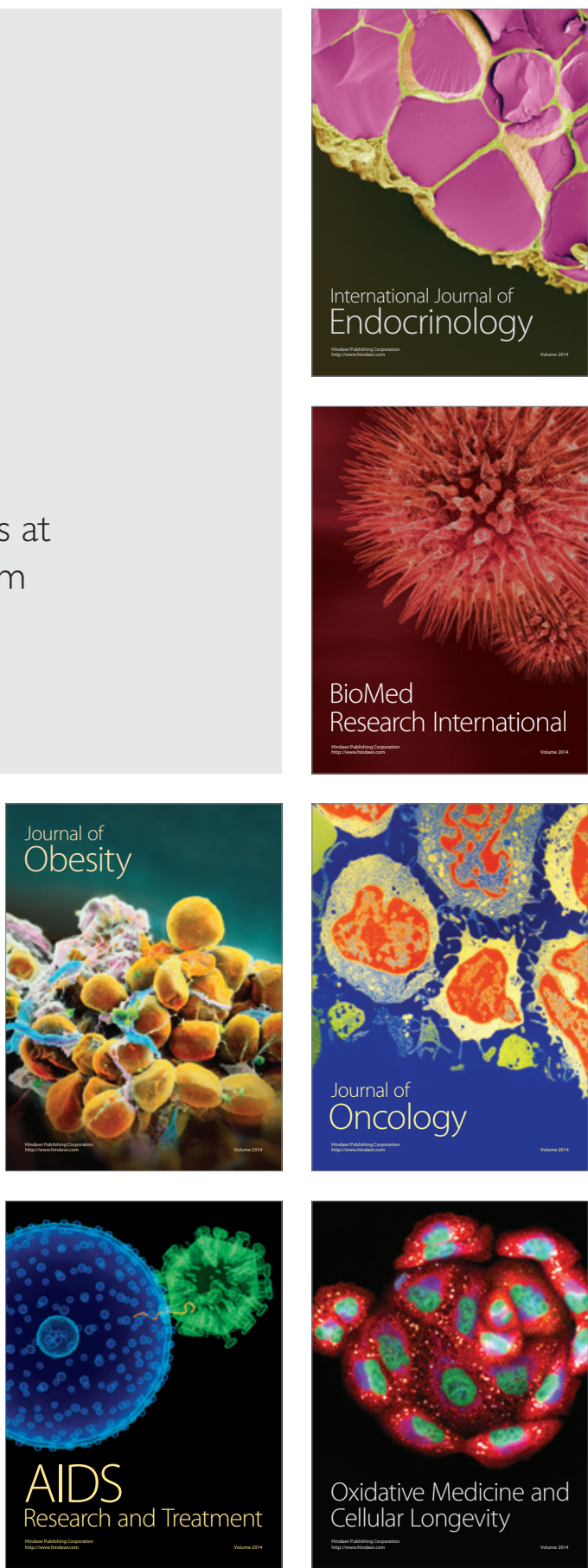